\title{
Java Memory Model aware Software Validation
}

\author{
Arnab De \\ Indian Inst. of Science \\ arnabde03@gmail.com
}

\author{
Abhik Roychoudhury \\ National Univ. of Singapore \\ abhik@comp.nus.edu.sg
}

\author{
Deepak D'Souza \\ Indian Inst. of Science \\ deepakd@csa.iisc.ernet.in
}

\begin{abstract}
The Java Memory Model (JMM) provides a semantics of Java multithreading for any implementation platform. The JMM is defined in a declarative fashion with an allowed program execution being defined in terms of existence of "commit sequences" (roughly, the order in which actions in the execution are committed). In this work, we develop OpMM, an operational under-approximation of the JMM. The immediate motivation of this work lies in integrating a formal specification of the JMM with software model checkers. We show how our operational memory model description can be integrated into a Java Path Finder (JPF) style model checker for Java programs.
\end{abstract}

\section{INTRODUCTION}

With increased push towards concurrent and parallel programming, developing tools and techniques for reliable concurrent programming is a must. Programmers tend to find parallel/concurrent programming harder than sequential programming - owing to the many possible program executions for any given program input. Moreover, programming languages like Java and C\# describe the semantics of multi-threading via a language level Memory Model. The language level memory model is somewhat synonymous to the semantics of multi-threading in the concerned programming language - it describes which writes can be visible to a program read operation. As a simple example, one may consider the following program fragment with shared variables A, B and local variables $r 1, r 2$, all initially 0 .

\begin{tabular}{l|l} 
Thread 1 & Thread 2 \\
\hline $\mathrm{A}=1 ;$ & $\mathrm{r} 1=\mathrm{B} ;$ \\
$\mathrm{B}=1 ;$ & $\mathrm{r2}=\mathrm{A} ;$
\end{tabular}

We would normally assume that at the end of the program we cannot have $r 1==1 \wedge r 2==0$ since $B$ is set after $A$ in Thread 1. However, in order to allow underlying compiler/hardware optimizations the language level memory model may allow re-ordering of the writes in Thread 1 , thereby making the result $r 1==1 \wedge$ $r 2==0$ possible at the end of the program. Such intricacies in

Permission to make digital or hard copies of all or part of this work for personal or classroom use is granted without fee provided that copies are not made or distributed for profit or commercial advantage and that copies bear this notice and the full citation on the first page. To copy otherwise, to republish, to post on servers or to redistribute to lists, requires prior specific permission and/or a fee.

PASTE '08, Atlanta, Georgia USA.

Copyright 2008 ACM 978-1-60558-382-2/08/11 ...\$5.00. the memory model makes formal reasoning about programs particularly difficult.

The Java Memory Model [12] essentially describes all allowed behaviors of a multi-threaded Java program on any implementation platform. The formal description of the Java Memory Model is declarative - it describes a notion of execution (a partial order of actions) and then defines what is an "allowed execution". However, the test of whether an execution is allowed is not directly executable. The model defines an execution as an allowed execution if there exists a commit sequence (sequence of sets of committing actions in the execution) satisfying certain properties. However, given an execution, even testing whether it is an allowed execution is non-trivial. It will require the construction of a sequence of commit-sets. Unfortunately, no algorithm on how to construct these commit sets is given in the Java Memory Model.

In this paper, we develop an operational approximation of the Java Memory Model (JMM) and use it for automated software validation via model checking. Our memory model is an underapproximation of the JMM in terms of allowed executions. We note that in (explicit-state) model checking we need to (a) traverse program executions, and (b) on-the-fly evaluate whether these are "allowed" executions. No efficient algorithm for construction and traversal of allowed program executions from the JMM is known. This is where our operational approximation fits in. We integrate our operational characterization of the JMM with an on-the-fly software model checker that checks for assertion violations. We call our operational Memory Model as OpMM. Since OpMM is a strict under-approximation of the JMM, an OpMM-aware model checker can be used to find bugs in a given program, rather than verify it.

Note that although the JMM guarantees Sequential Consistency (SC) for race-free programs [12], programs in general may have races for high performance or other reasons. A memory model aware model checker like ours is most suitable for validating programs which are not proven to be race-free.

\section{BACKGROUND AND RELATED WORK}

Memory models have been used in shared memory multiprocessors. Details of hardware memory models appear in $[1,5]$.

The interest in programming language memory models is relatively new. Among the different programming languages, work on the Java Memory model (JMM) has received the most attention. A formal description of the JMM has been presented in [7, 12] with inputs from researchers and developers alike. This model is presented in a non-operational, declarative style. No procedure for efficiently checking (say in polynomial time) whether a given execution of a given program is an allowed behavior is known.

Program validation in a memory model aware fashion was first studied in the context of hardware memory models. Park and Dill 
[13] developed executable memory models for SPARC architectures and used them to verify synchronization routines. Gopalakrishnan et. al. [8] have used SAT solving to check whether an execution is allowed by a multi-processor memory model, in particular the Intel Itanium memory model. In recent works, Burckhardt, Alur and Martin [3] study bounded model checking of concurrent data types under relaxed hardware memory models.

In our past work [10], we have developed a bytecode level invariant checker for $\mathrm{C \#}$, which proceeds in a memory model aware fashion. This required us to formally specify the $\mathrm{C \#}$ memory model and integrate inside a bytecode level model checker. However, the C\# memory model is simpler than the JMM, and it can be specified as a re-ordering table describing which pairs of operations can be re-ordered. As we will describe now, the JMM specification is more complex, and cannot be captured by a re-ordering table. Hence our first task is to develop an operational version of the JMM, which we then use for bytecode level model checking.

\section{OVERVIEW OF OUR APPROACH}

We now describe the JMM and OpMM models via several examples. As our first example, we present the following program fragment, where $r 1-r 6$ are locals, $p, q$ are shared variables and initially $\mathrm{p}==\mathrm{q}$ and $\mathrm{p} \cdot \mathrm{x}==0$ :

\begin{tabular}{ll} 
Thread 1: & Thread 2: \\
\hline$r 1=p ;$ & $r 6=p ;$ \\
$r 2=r 1 . x ;$ & $r 6 . x=3 ;$ \\
$r 3=q ;$ & \\
$r 4=r 3 . x ;$ & \\
$r 5=r 1 . x ;$ &
\end{tabular}

Figure 1: Example 1

A compiler optimization might replace the last statement of Thread 1 by $r 5=r 2$; as they are assigned same expression $r 1 . x$ and the value of the expression is not changed in Thread 1. But this optimization may lead to the behavior $r 2==r 5==0, r 4==$ 3 after the execution, which is not allowed by Sequential Consistency on the original program.

In order to support such optimizations, JMM allows this behavior. In the resulting execution, the reads into $r 2$ and $r 5$ see the write of the initial value and the read into $r 4$ sees the write by Thread 2. The commit sequence for validating this execution (as required by JMM [12]) is the following. In the first step, we can commit all actions except $r 4=r 3 . x$. As all non-committed reads must see a write that happens-before it, the read into $r 4$ sees the initial write of value 0 . In the next step, the read into $r 4$ is committed, but it still gets the value 0 as the committing reads must see a write which is committed earlier as well as happens-before the read. In the next step, when the read is committed at least one step earlier, it can see any write that has been committed before (but not necessarily happens-before) - hence the read now can see the write $r 6 . x=3$ from Thread 2, resulting in the desired behavior.

As mentioned, validating an execution in JMM requires finding a commit sequence according to the rules given in [12]. There is no efficient algorithm for this purpose other than generating and testing all possible commit sequences. The problem of generating all legal executions of a given Java program is even more difficult.

Now we show how we can generate an execution in OpMM that displays the desired behavior in the program of Figure 1. In OpMM, actions are executed in a total-order consistent with the program-order, but a non-volatile read can see any previous write in that total order that is happens-before consistent (thus differing from SC). To achieve this, a state in OpMM consists of different views of the heap, one by each thread, instead of a single global heap. Each location of the heap contains a set of values which a read is allowed to see. In this example, in an execution where the write to $r 6 . x$ in Thread 2 is scheduled before the last two reads of Thread 1, the heap location for $r 3 . x$ and $r 1 . x$ contains both the values 0 and 3 . Hence it is possible for $r 4$ to read the value 3 and $r 5$ to read the value 0 , and thus producing the desired behavior.

OpMM considers synchronizations while deciding the writes that should not be seen by a later read (in order to maintain happensbefore consistency, as required by the JMM). Let us consider the following example where $r 1$ is a local and $x, 1$ are shared variables, $\mathrm{x}$ initialized to 0 and $\mathrm{I}$ is initially unlocked.

\begin{tabular}{ll} 
Thread $1:$ & Thread 2: \\
\hline lock $1 ;$ & lock $1 ;$ \\
$\mathrm{x}=1 ;$ & $\mathrm{x}=2 ;$ \\
r1 $=\mathrm{x} ;$ & unlock $1 ;$ \\
unlock $1 ;$ &
\end{tabular}

Figure 2: Example 2

In any execution of this program, the only value $r 1$ can get is 1, from the write in Thread 1. In any execution in OpMM, two cases might happen: Thread 1 can execute entirely before Thread 2 or vice versa. No other interleaving is possible as OpMM obeys mutual exclusion of lock operations. In the first case, the write $\mathrm{x}$ $=1$ masks the initial value of $x$ (i.e., 0 ) as in the same thread, a newer write masks the older one. Hence when the read $r 1=$ $\mathrm{x}$ is executed, only one value 1 can be seen. In the second case, when Thread 1 locks the same monitor unlocked by Thread 2, their views of the heap are synchronized. Consequently the write of 1 in Thread 1 masks the write by Thread 2 as well as the initial value of $\mathrm{x}$, resulting in the desired behavior.

It should be noted that OpMM is a strict under-approximation of JMM i.e. there are executions which are allowed by JMM but not by OpMM. Let us consider the following example where all variables are initialized to 0 .

\begin{tabular}{ll} 
Thread 1: & Thread 2: \\
\hline $\mathrm{r} 1=\mathrm{x} ;$ & $\mathrm{r} 2=\mathrm{y} ;$ \\
$\mathrm{y}=1 ;$ & $\mathrm{x}=1 ;$
\end{tabular}

Figure 3: Example 3

The behavior $r 1==r 2==1$ after the execution is allowed by JMM [12], but it is not allowed in OpMM. To allow this behavior, the read of $x$ to see the write $x=1$; and the read of $y$ needs to see the write $\mathrm{y}=1$; . There is no total-order consistent with the program-order where both the writes occur before the corresponding reads. As a result, it becomes difficult to model such behaviors operationally because it loses a clear notion of trace - a sequence of actions that modify the states. Hence OpMM does not model the entire JMM.

\section{DESCRIPTION OF OPMM}

\subsection{Programs}

The programming language we consider is an abstract version of the Java bytecode. Local variables are of the form $r n$. The shared variables can be accessed through (static or non-static) field access of a reference type local variable. $C, f, v$ denote any class, field, volatile field, respectively. All statements are prefixed with a thread id while describing the semantics (written as $t: c$ ). Threads, classes, objects, monitors and volatiles have unique ids.

Statements. The statements we are considering are one of the following forms: (1) Writing to shared variables: $r i . f=r j$, (2) Reading from shared variables: $r i=r j \cdot f$, (3) Writing to volatile variables: $r i . v=r j$, (4) Reading from volatile variables: $r i=r j \cdot v$, (5) Locking a monitor: lock $r i$, (6) Unlocking a monitor: unlock $r i$, (7) Creating an object: $r i=$ new 
$C$, (8) Starting a thread: start $r i$, (9) Interrupting a thread, (10) Determining whether a thread is interrupted, (11) Detecting whether a thread is alive, (12) skip, (13) Assignment statements involving only local variables, (14) Control statements, (15) Method calls and returns, and (16) Throwing and catching of exceptions. In this paper we concentrate on statements of type $1-11$ as they are relevant to the memory model. Other statements must follow the intra-thread semantics as defined in [9].

Program. The program consists of one or more threads. Each thread has one or more methods. There is one thread with main method (referred as main thread hereafter). Other threads must have a run method.

Structure of the Heap. The heap can be seen to be made of object areas, each object area allocated to a particular object. As Java is a strongly typed language, reference type local variables point to one of these object areas or null. The object areas are divided into cells, one cell for each field of the object allocated in that object area. The cells do not contain a value, but a writelist. A write-list can contain three types of elements: (i) write-item (WI) which is a $\langle$ value, tid $\rangle$ pair, (ii) a release-item (RI) which is a $\langle$ lock, tid $\rangle$ pair or a $\langle$ volatile, tid $\rangle$ pair, and (iii) an acquire-item (AI) which is again a $\langle$ lock, tid $\rangle$ pair or a $\langle$ volatile, $t i d\rangle$ pair.

\subsection{Structure of States and Transitions}

Program State. The program state (denoted by $\Omega$ ) consists of one local state for each active thread, and a global state for monitors and volatiles. Each local state $L_{t}(t$ is the corresponding $t i d$ ) consists of the following parts:

- A stack of frames, one frame for each outstanding method call in the thread (as mentioned in [11]), denoted by $S_{t}$. Each frame, among other things (as specified in The Java Virtual Machine Specification [11]), contain values of the local variables of the corresponding method. We denote the local variable map of the top frame by $\sigma_{t}$.

- A view of the heap (denoted by $H_{t}$ ) which is a function from cells to a write-list, as specified in Section 4.1.

The global state consists of monitor states and volatile states. The monitor state is a mapping $M$ from monitors to tuples of the form $\langle$ tid, lockcount $\rangle$. When the lockcount is 0 , the tid part also has a value 0 (we assume that there is no thread with tid 0 ). The volatile state $V$ is a mapping from volatile ids to the value of the corresponding volatile variable. It should be noted that our notion of program state has no global heap, except for the values of volatiles and monitors which are globally maintained. The state might contain other information like thread status, class information for objects etc which are required by Java Virtual Machine [11]. As this information is not directly related to the memory model, we do not include it in our description here.

State Transitions. A program statement can cause state transitions. If a statement $c$ changes the program state from $\Omega$ to $\Omega^{\prime}$, we write it as $\langle c, \Omega\rangle \rightarrow \Omega^{\prime}$. We did not include program counter $(p c)$ in our description of states as that is not relevant for our work. Execution order of instructions is described informally in Section 4.3.1.

\subsection{Operational Semantics}

We present the operational semantics of OpMM which is a sound but incomplete approximation of JMM. The semantics is given in form of inference rules. The consequence of any of these rules is a state transition by a statement. The premises are the conditions that must be satisfied to enable the transition given as consequence.

\subsubsection{Restrictions on Execution Order.}

The execution should start at the ma in method of the main thread. At each step, only one statement is executed from the active threads, if the statement is enabled according to the semantics as given in Section 4.3.2, producing a total-order of actions. Statements from a thread are executed in program order. Statements from threads other than main can by executed only after they are started (using a start statement). Executions in other threads should start at the run method of the corresponding thread.

\begin{tabular}{l}
\hline Algorithm 1 Mask\&Read \\
Input: write-list wl, thread-id tid \\
Output: Set of write-items ws
\end{tabular}

/*Lock id, Vol id and Tid are the sets of lock ids, volatile ids and thread ids*/

WriteSet: Set of write-items

ThSet: Tid $\rightarrow\{$ marked, unmarked, undef $\}$

AcSet: (Lock id $\cup$ Vol id) $\rightarrow$ \{marked, unmarked, undef $\}$

1: WriteSet $\leftarrow \emptyset$

2: $\forall k \in$ Lock id $\cup$ Vol id: AcSet $(k) \leftarrow$ undef

3: $\forall t \in$ Tid: ThSet $(t) \leftarrow$ undef

4: ThSet $($ tid $) \leftarrow$ unmarked

5: for all element e in wl, starting from the newest do

6: $\quad$ if $e$ is a write-item $\langle v, t\rangle$ then

7: $\quad$ if $\operatorname{ThSet}(t)=$ undef then

8: $\quad$ WriteSet $\leftarrow$ WriteSet $\cup\{\langle v, t\rangle\}$

9: $\quad$ else if $\operatorname{ThSet}(t)=$ unmarked then

10: $\quad$ WriteSet $\leftarrow$ WriteSet $\cup\{\langle v, t\rangle\}$

11: $\quad T h \operatorname{Set}(t) \leftarrow$ marked

12: $\quad$ else if $\operatorname{ThSet}(t)=$ marked then

13: $\quad$ skip

14: $\quad$ end if

15: $\quad$ else if $e$ is an acquire-item $\langle k, t\rangle$ then

16: $\quad$ if $\operatorname{AcSet}(k)=$ marked or ThSet $(t)=$ undef then

17: skip

18: $\quad$ else

19: $\quad \operatorname{AcSet}(k) \leftarrow \operatorname{ThSet}(t)$

20: $\quad$ end if

21: $\quad$ else if $e$ is a release-item $\langle k, t\rangle$ then

22: $\quad$ if $T h S e t(t)=$ marked $\operatorname{or} A c S e t(k)=$ undef then

23: $\quad$ skip

24: else

25: $\quad \operatorname{ThSet}(t) \leftarrow \operatorname{AcSet}(k)$

26: end if

27: end if

28: end for

29: $w s \leftarrow$ WriteSet

30: return $w s$

\subsubsection{Semantics of Statements.}

We now present the operational semantics rules of OpMM for the different language constructs of Java. The following conventions are used while describing the semantics for the sake of simplicity: In the inference rules, $k$ ranges over thread ids and $h$ ranges over cells in the universal quantifiers. Field access of an object returns the corresponding cell. $\Omega[A \rightarrow B]$ denotes a state same as $\Omega$ but the component $A$ has been changed to $B . \Omega[F \mid x: v]$ is a shorthand expression representing a state which is equivalent to $\Omega$ except $F(x)$ has a new value $v$. Here we give a formal semantics 
(Wr)

$$
\frac{v=\sigma_{t}(\mathrm{rj}) \quad h=\sigma_{t}(\mathrm{ri}) . \mathrm{f} \quad \Omega^{\prime}=\Omega\left[\forall k: H_{k}(h) \rightarrow \operatorname{Append}\left(H_{k}(h), \mathrm{WI}(\langle v, t\rangle)\right)\right]}{\langle t: \mathrm{ri} . \mathrm{f}=\mathrm{rj}, \Omega\rangle \rightarrow \Omega^{\prime}}
$$

$(\mathrm{Rd})$

$$
\frac{\left\langle v, t^{\prime}\right\rangle \in M a s k \& \operatorname{Read}\left(H_{t}\left(\sigma_{t}(\mathrm{rj}) . \mathrm{f}, t\right)\right)}{\langle t: \mathrm{ri}=\mathrm{rj} . \mathrm{f}, \Omega\rangle \rightarrow \Omega\left[\sigma_{t} \mid \mathrm{ri}: v\right]}
$$

(U1)

$$
\begin{gathered}
M\left(\sigma_{t}(\mathrm{ri})\right)=\langle t, n\rangle \\
\Omega^{\prime}=\Omega\left[\forall k, \forall h: H_{k}(h) \rightarrow \operatorname{Append}\left(H_{k}(h), \operatorname{RI}\left(\left\langle\sigma_{t}(\mathrm{ri}), t\right\rangle\right)\right) ; M \rightarrow \operatorname{Unlock}\left(\sigma_{t}(\mathrm{ri})\right)\right]
\end{gathered}
$$

$$
\begin{gathered}
M\left(\sigma_{t}(\mathrm{ri})\right)=\langle 0,0\rangle \\
\Omega^{\prime}=\Omega\left[\forall k, \forall h: H_{k}(h) \rightarrow \operatorname{Append}\left(H_{k}(h), \operatorname{AI}\left(\left\langle\sigma_{t}(\mathrm{ri}), t\right\rangle\right)\right) ; M \rightarrow \operatorname{Lock}\left(\sigma_{t}(\mathrm{ri}), t\right)\right]
\end{gathered}
$$

$$
\begin{gathered}
M\left(\sigma_{t}(\mathrm{ri})\right)=\langle t, n\rangle \\
\Omega^{\prime}=\Omega\left[\forall k, \forall h: H_{k}(h) \rightarrow \operatorname{Append}\left(H_{k}(h), \operatorname{AI}\left(\left\langle\sigma_{t}(\mathrm{ri}), t\right\rangle\right)\right) ; M \rightarrow \operatorname{Lock}\left(\sigma_{t}(\mathrm{ri}), t\right)\right]
\end{gathered}
$$

$(\mathrm{V}-\mathrm{Wr})$

$(\mathrm{V}-\mathrm{Rd})$

$$
\frac{v=\sigma_{t}(\mathrm{rj}) \quad \Omega^{\prime}=\Omega\left[\forall k, \forall h: H_{k}(h) \rightarrow \operatorname{Append}\left(H_{k}(h), \operatorname{RI}\left(\left\langle\sigma_{t}(\mathrm{ri}) \cdot \mathrm{v}, t\right\rangle\right)\right)\right]}{\langle t: \mathrm{ri} \cdot \mathrm{v}=\mathrm{rj}, \Omega\rangle \rightarrow \Omega^{\prime}\left[V \mid \sigma_{t}(\mathrm{ri}) \cdot \mathrm{v}: v\right]}
$$

$$
\frac{v=V\left(\sigma_{t}(\mathrm{rj}) \cdot \mathrm{v}\right) \quad \Omega^{\prime}=\Omega\left[\forall h: H_{t}(h) \rightarrow \operatorname{Append}\left(H_{t}(h), \operatorname{AI}\left(\left\langle\sigma_{t}(\mathrm{rj}) \cdot \mathrm{v}, t\right\rangle\right)\right)\right]}{\langle t: \mathrm{ri}=\mathrm{rj} \cdot \mathrm{v}, \Omega\rangle \rightarrow \Omega^{\prime}\left[\sigma_{t} \mid \mathrm{ri}: v\right]}
$$

Figure 4: OpMM rules for read/write, lock/unlock and volatile read/write statements

of the statements relevant to the memory model. Other statements follow the intra-thread semantics in [9].

Write statement. A write statement appends the write to the write-list for the corresponding cell of every local state. Rule (Wr) in Figure 4 is the operational semantics rule for the write statement. Here Append $(l, i)$ appends the element $i$ to the list $l$.

Read statement. A read statement updates the local variable with a value from the set of write-items returned by the Mask\&Read function applied on the corresponding local write-list and the reading thread-id. Rule (Rd) in Figure 4 is the operational semantics rule for the read statement. Note that the Mask\&Read function is defined as an algorithm in Algorithm 1. The main function of the algorithm is to prevent a read $r$ from seeing a write $w$ if there is another write $w^{\prime}$ such that $w \stackrel{h b}{\longrightarrow} w^{\prime} \stackrel{h b}{\longrightarrow} r$ where $h b$ is the happensbefore relation as defined in [12]. Informally, it maintains two functions: ThSet and AcSet maps the thread ids and synchronization object ids (locks/ volatiles) respectively to undef, unmarked or marked. Whenever a thread $t$ is not synchronized with the input thread, $\operatorname{ThSet}(t)=$ undef. Similarly when a volatile/monitor $k$ is not acquired by any thread $t$ in ThSet (i.e. ThSet $(t) \neq$ undef), the Mask\&Read algorithm treats it as $A c S e t(k)=$ undef. Algorithm 1 initially puts the input thread id into ThSet as unmarked and traverses the input write-list, starting from the newest item. When it finds an acquire-item whose thread id belongs to ThSet, it puts the lock/ volatile id into the AcSet with the same marking. Similarly, when it sees a release-item whose lock/ volatile id belongs to AcSet, it puts the thread id into the ThSet with the same marking. A write is put into the write-set if the thread id is unmarked or undef in ThSet. If the thread id is unmarked, it is marked after seeing the write. If a thread id is already marked, the writes by that thread are not put into write-set.

Unlock statement. An unlock statement can execute only when the executing thread holds the lock. It appends the corresponding release-item to all the write-lists. It also changes the monitor state. Rule (Ul) in Figure 4 is the operational semantics rule for the unlock statement. Here $\operatorname{Unlock}(m)$ reduces the lockcount of $M(m)$ and if it reaches 0 , changes the tid of $M(m)$ to 0 . Recall that $M$ is a mapping from monitors to the threads locking a monitor.

Lock statement. The lock statement can execute if the corresponding monitor is not locked or locked by the same thread. It appends the corresponding acquire-item to all the write-lists, and changes the monitor state. Rules (L-1) and (L-2) in Figure 4 are the rules for the lock statement. Here $\operatorname{Lock}(m, t)$ increments the lockcount of $M(m)$ and if was 0 , changes the tid of $M(m)$ to $t$.

Volatile write statement. Volatile writes directly update the global state for volatiles. It also appends the corresponding releaseitem to each write-list of each local state. Rule (V-Wr) in Figure 4 is the operational semantics rule for the volatile write statement.

Volatile read statement. Volatile reads read the value directly from the global state of volatiles and update the local variable. It also appends the corresponding acquire-item to each write-list of each local state. Rule (V-Rd) in Figure 4 is the operational semantics rule for volatile reads. 
Object creation statement. The object creation statement $r i$ $=$ new $\mathrm{C}$ creates a new object area, extending $H_{k}$ for all threads $k$. A unique id is assigned to it. The local map for $r i$ is updated to hold reference to the new object. After the object is created, the constructor call can be treated as a normal method call.

Thread creation statement. When a thread $t^{\prime}$ is spawned by a thread $t$, the spawned thread inherits the object areas from the spawning thread, i.e. $H_{t^{\prime}}$ becomes equal to $H_{t}$. Moreover, all the write-lists of the state are appended by a release-item $\langle l, t\rangle$ and an acquire-item $\left\langle l, t^{\prime}\right\rangle$, in that order, where $l$ is a unique lock id that does not occur in the execution.

Thread termination and interruption. When a thread $t$ is terminated, then after modification of the local state (such as thread status), a release item $\langle l, t\rangle$ is appended to all the write-lists. When an action from some other thread $t^{\prime}$ detects that $t$ has terminated, an acquire-item $\left\langle l, t^{\prime}\right\rangle$ is appended to all the write-lists, before any other modification in the state by that action. $l$ is a unique lock id that does not occur in the execution. Similar steps are followed for thread interruption.

\section{EXPRESSIVE POWER OF THE MODEL}

In this section, we show that the memory model semantics proposed in Section 4.3 is an under-approximation of the Java Memory Model in terms of allowed executions. We have also shown that OpMM is strictly weaker than the hardware memory model TSO — details appear in [6].

\subsection{Traces and Occurs-Before Relation}

A program trace allowed by the proposed semantics is an interleaving of the actions $c_{0}, \ldots c_{n}$ such that

$$
\Omega_{0} \stackrel{c_{0}}{\rightarrow} \Omega_{1} \ldots \Omega_{n} \stackrel{c_{n}}{\rightarrow} \Omega_{n+1}
$$

where $\Omega_{0}$ is an initial state. The order of execution must obey the restrictions given in Section 4.3.1 and each transition $\left\langle c_{j}, \Omega_{j}\right\rangle \rightarrow$ $\Omega_{j+1}$ must be allowed by the semantics given in Section 4.3.2.

Given a trace $t$, we say $c_{i} \stackrel{o b}{\longrightarrow} c_{j}$, if $c_{i}$ appears before $c_{j}$ in the trace $t$. We call $\stackrel{o b}{\rightarrow}$ as the occurs-before relation

\subsection{Construction of an Execution from a Trace}

Given a trace (as defined in Section 5.1), we first need to construct an execution (according to the definition of execution given in [12]) corresponding to it.

The execution is $E=\langle P, A, \stackrel{p o}{\rightarrow}, \stackrel{s o}{\rightarrow}, W, V, \stackrel{s w}{\rightarrow}, \stackrel{h b}{\rightarrow}\rangle$ where $P$ is the program, $A$ is the set of actions in the trace, $a \stackrel{p o}{\rightarrow} b$ if they belong to the same thread and $a \stackrel{o b}{\longrightarrow} b, a \stackrel{s o}{\longrightarrow} b$ if both of them are synchronization actions and $a \stackrel{o b}{\longrightarrow} b, W(a)=b$ if $a$ is a read action and it reads a write by action $b$ from its write-list, $V(a)$ is the value written by a write action $a$, the synchronizes-with relation $\stackrel{s w}{\longrightarrow}$ and the happens-before $\stackrel{h b}{\rightarrow}$ are as defined as in [12]. For example, an unlock action on a monitor $m$ synchronizes with all "subsequent" (as per the synchronization order relation $\stackrel{s o}{\rightarrow}$ ) lock actions on $m$ that were performed on any thread. A write to a volatile variable $u$ synchronizes-with all "subsequent" (as per the synchronization order relation $\stackrel{s o}{\rightarrow}$ ) reads of $u$ performed by any thread. The happens-before relation is the transitive closure of program order and synchronizes-with order, that is, $\stackrel{h b}{\longrightarrow}=(\stackrel{p o}{\longrightarrow} \cup \stackrel{s w}{\longrightarrow})^{*}$. It should be noted that $\stackrel{p o}{\longrightarrow}$ is a total order among the actions from the same thread and $\stackrel{s o}{\longrightarrow}$ is a total order among synchronization actions, as required by JMM. If a trace allowed by OpMM leads to a state that violates an assertion, the corresponding execution constructed from the trace will also violate the assertion. As our notion of underapproximation is based on allowed executions, it is enough to show that the execution constructed from the trace is allowed by JMM.

\subsection{Some Properties of OpMM}

We now establish some key properties of the OpMM model, which will be useful for establishing that OpMM is a strict underapproximation of the JMM.

THEOREM 1 . If $a \stackrel{h b}{\longrightarrow} b$ in the constructed execution, then $a \stackrel{o b}{\longrightarrow}$ $b$ in the trace.

PROOF. As $\stackrel{h b}{\longrightarrow}=(\stackrel{p o}{\longrightarrow} \cup \stackrel{s w}{\longrightarrow})^{*}$, it is enough to show that $p o$ and $s w$ of the constructed execution are consistent with the $o b$ relation of the trace. If $a \stackrel{p o}{\rightarrow} b$, then by construction of $p o$ edges (in Section 5.2), $a \stackrel{o b}{\rightarrow} b$. Let $a \stackrel{s w}{\longrightarrow} b$. Then either $a \stackrel{p o}{\longrightarrow} b$ or $a \stackrel{s o}{\longrightarrow} b$ or $a$ changes the thread status which is observed by $b$. As all of these relations are consistent with $o b, s w$ is also consistent with $o b$.

LEMMA 2. During the execution of Mask\&Read algorithm (Algorithm 1), for all tid t and sync id (lock id or volatile id) $l$, ThSet $(t)$ and $A c S e t(l)$ can change their values from undef to unmarked to marked, but in no other order.

This can be proved by induction on the number of iterations of the main loop of Alg. 1.

LEMMA 3. Let $t^{\prime}: a \stackrel{h b}{\rightarrow} t: r$ where $a$ is an action from thread $t^{\prime}, r$ is a read action from thread $t$ and the result of a is present in the write-list for $r$ in $H_{t}$. Then after a is encountered during the execution of Mask\&Read algorithm for $r$ (Algorithm 1), ThSet $\left(t^{\prime}\right) \neq$ undef.

PROOF. We have $t^{\prime}: a \stackrel{h b}{\rightarrow} t: r$ and $\stackrel{h b}{\rightarrow}=(\stackrel{p o}{\longrightarrow} \cup \stackrel{s w}{\rightarrow})^{*}$. We prove by induction on number of $p o$ or $s w$ edges between $a$ and $r$.

Base case: If the number of edges is one, then $t: a \stackrel{p o}{\rightarrow} t: r$ (as $r$ cannot be target of an $s w$ edge). ThSet $(t)$ is set to unmarked in line 4. By Lemma 2, it cannot change to undef later when we encounter $a$.

Induction step: By induction hypothesis, if $\hat{t}: \hat{a} \stackrel{h b}{\rightarrow} r$ using $n$ po or $s w$ edges, then after encountering $\hat{a}, \operatorname{ThSet}(\hat{t}) \neq$ undef. If $a \stackrel{p o}{\rightarrow} \hat{a}$, then $t^{\prime}=\hat{t}$, hence proved. Otherwise, if $a \stackrel{s w}{\rightarrow} \hat{a}, a$ is a release-item and corresponding AcSet is not undef (as ThSet $(\hat{t}) \neq$ undef while encountering $\hat{a})$. Hence by lines $22-26, \operatorname{ThSet}\left(t^{\prime}\right) \neq$ undef after encountering $a$.

COROLlary 4. If $t^{\prime}: w \stackrel{h b}{\rightarrow} t: r$, where $w$ is a write action to the same location as read $r$, then before $w$ is encountered during the execution of Mask\&Read algorithm for $r$ (Algorithm 1), $\operatorname{ThSet}\left(t^{\prime}\right) \neq$ undef.

The proof follows from Lemma 3 and the fact that the last edge in the happens-before path will always be $p o$ in case of a write.

LEMMA 5. If $t_{1}: w_{1} \stackrel{h b}{\rightarrow} t_{2}: w_{2} \stackrel{h b}{\rightarrow} t: r$, where $w_{1}$ and $w_{2}$ are writes to the same location as read $r$ then before $w_{1}$ is encountered during the execution of Mask\&Read algorithm for $r$ (Algorithm 1), $\operatorname{ThSet}\left(t_{1}\right)=$ marked .

PROOF. $w_{2}$ will be encountered before $w_{1}$. After $w_{2}$ is encountered, by Lemma 4 and lines $9-13$ of Algorithm 1, ThSet $\left(t_{2}\right)=$ marked. Using Lemma 2, by induction on the number of po and $s w$ edges between $w_{1}$ and $w_{2}$, this Lemma can be proved. 
THEOREM 6. If $t_{1}: w_{1} \stackrel{h b}{\longrightarrow} t_{2}: w_{2} \stackrel{h b}{\longrightarrow} t: r$, where $w_{1}$ and $w_{2}$ are writes to the same location as read $r$, then $w_{1} \notin w s$ (returned by Mask\&Read algorithm (Algorithm 1)) executed during processing of $r$.

This follows directly from Lemma 5 and lines 12 - 13 of the Algorithm 1.

\subsection{Well-formedness of Executions}

Using the above properties we can show that our executions are well-formed as per the JMM [12]. Details of these arguments appear in [6]. The key to our argument is showing that any execution obeys happens-before consistency. It can be shown by contradiction. Two cases can happen:

(A) There is a r such that $r \stackrel{h b}{\longrightarrow} W(r)$. In our model, if $r \stackrel{h b}{\longrightarrow} W(r)$, then we have $r \stackrel{o b}{\rightarrow} W(r)$ in the trace (by Theorem 1). But this means when $r$ was executed, $W(r)$ was not part of the write-list, implying $r$ cannot read $W(r)$.

(B) There is a r such that $W(r) \stackrel{h b}{\rightarrow} w \stackrel{h b}{\rightarrow} r$. This is not possible by Theorem 6.

\subsection{Causality Requirements}

Finally, we need to show that the executions generated by our model meet the causality requirements given in JMM (see Section 4.5 of the JMM paper [12]). For this purpose, we need to find set of actions to commit in each step and a validating execution for each of them. We can simply follow the occurs-before order of the actions of the thread. We commit the instructions in that order and use the execution up to that point to validate it. It must be noted that when we commit a read, in that step, the read sees a write that happens before it (there is at least one such write due to initialization), but in the next step we can change it to the write it finally sees as that write has already been committed.

This completes the proof of the fact that OpMM is a strict underapproximation of the JMM.

\subsection{Disallowed Behavior}

Note that OpMM is a strict under-approximation of JMM. This naturally raises the following question: what kind of program behaviors are allowed by the JMM, but disallowed by OpMM? We address this issue in the following.

A write-seen edge (denoted as ws) is an edge from a write action $w$ to a read action $r$ such that $W(r)=w$. In some executions allowed by JMM, the happens-before and write-seen edges form a cycle. For example, in the example of Fig. 3, there is a cycle $\mathrm{r} 1=\mathrm{x} ; \stackrel{h b}{\rightarrow} \mathrm{y}=\mathrm{r} 1 ; \stackrel{w s}{\rightarrow} \mathrm{r} 2=\mathrm{y} ; \stackrel{h b}{\rightarrow} \mathrm{x}=\mathrm{r} 2 ; \stackrel{w s}{\rightarrow} \mathrm{r} 1=\mathrm{x} ;$

As $a_{1} \stackrel{h b}{\rightarrow} a_{2}$ and $a_{1} \stackrel{w s}{\longrightarrow} a_{2}$ both imply $a_{1} \stackrel{o b}{\longrightarrow} a_{2}$ and $o b$ is a partial order, such cycles are not allowed in OpMM. As a result, any execution containing such cycles are disallowed in OpMM.

\section{EXPERIMENTS}

We have integrated OpMM into a bytecode level model checker for Java. This allows us to use OpMM for property checking of Java programs. This clearly goes beyond conventional software validation, which ignores the language level memory model, and implicitly assume Sequential Consistency as the execution model.

Implementation issues. Our model checker is an explicit state on-the-fly model checker in the style of the Java Path Finder (JPF) [15]. It takes the bytecode representation of a program as input and detects whether any assertion specified in the program can be violated under OpMM. As the state space of the input program can be infinite, we do not guarantee termination in general, but a depthbound can be imposed on the depth-first search to force termination. While integrating OpMM to a model checker, we observe that in OpMM the write-lists can grow unboundedly and thus, the size of a state can also grow unboundedly. We developed a technique to prune the write-lists in many cases (but not all). We modify the Mask\&Read algorithm (presented in Algorithm 1) to delete the write-items if the corresponding ThSet value is marked when the item is encountered during the traversal of the write-list. We also delete the release-items and acquire-items if the corresponding ThSet value is marked after processing of the items. The modified algorithm appears in [6], where we also prove that the modified algorithm returns the same results as Algorithm 1 (as a result of which the proof that OpMM is a strict under-approximation of JMM is unaffected).

Results. We present the results of running our tool on some subject programs in Table 1. For each subject program we check one invariant assertion encoding the correctness of the program. The subject program dcl is the original version of double-checked locking [14]. This program fragment is used for efficient lazy instantiation of a singleton class. Double-Checked Locking is a program fragment for instantiation in which (a) only one instance is generated, and (b) the instance is generated only on-demand. The assertion checks whether the singleton object reference is correctly constructed after lazy initialization. This assertion can be violated under JMM [2]. Our tool successfully detects the bug.

The subject program dcl-vol is the version of double-checked locking where the singleton object reference is declared volatile. This version works correctly under JMM, and our tool validates that it works correctly under OpMM. In other words, we check that the singleton object is properly constructed before it is referenced. The subject program singleton represents the same singleton pattern as $\mathrm{dcl}$, but here, instead of using double-checked locking, the lock is taken every time the reference is requested. This version works correctly under JMM and our tool validates it under OpMM.

Subject programs peterson and dekker are traditional algorithms for establishing mutual exclusion. The assertion checks whether mutual exclusion property of these programs can be violated. Both of them fail under JMM. Our tool detects the assertion violations.

\begin{tabular}{|l|r|r|r|r|}
\hline Program & \#bytecode & \#threads & $\begin{array}{r}\text { time } \\
(\mathrm{sec})\end{array}$ & $\begin{array}{r}\text { \#states } \\
\text { explored }\end{array}$ \\
\hline \hline dcl & 70 & 2 & 0.22 & 87 \\
\hline dcl-vol & 70 & 2 & 0.25 & 267 \\
\hline singleton & 67 & 2 & 0.27 & 297 \\
\hline peterson & 108 & 2 & 0.22 & 99 \\
\hline dekker & 135 & 2 & 0.23 & 101 \\
\hline
\end{tabular}

Table 1: Experimental Results

Table 1 shows the results of our experiments. All experiments were done on a $1.6 \mathrm{GHz}$ dual core machine with $1 \mathrm{~GB}$ main memory. The time reported is in seconds. In cases where the assertion is violated, the first counterexample is reported. Otherwise, the entire state space is exhausted.

Our checker is currently a prototype. In future, there exists scope for (a) optimizing the checker's implementation, and (b) reducing the visited state space by methods like partial order reduction [4].

\section{Acknowledgments}

This work was partially supported by NUS research grant R-252000-312-112, an A*STAR grant R-252-000-258-305 under EHS2 programme, and a Microsoft Research travel grant. 


\section{REFERENCES}

[1] S.V. Adve and K. Gharachorloo. Shared memory consistency models: A tutorial. IEEE Computer, pages 66-76, Dec. 1996.

[2] D. Bacon et al. The "double-checked locking is broken" declaration.

http: //www.cs.umd.edu/ pugh/java/

memoryModel/DoubleCheckedLocking . html.

[3] S. Burckhardt, R. Alur, and M. Martin. Checkfence:

Checking consistency of concurrent data types on relaxed memory models. In PLDI, 2007.

[4] E. Clarke, O. Grumberg, and D. Peled. Model Checking. MIT Press, 1999.

[5] D.E. Culler and J. Pal Singh. Parallel Computer Architecture: A Hardware/Software Approach. Morgan Kaufmann Publishers, 1998.

[6] A. De, A. Roychoudhury, and D. D'Souza. Java memory model aware software verification. Technical report, 2008. http: //clweb.csa.iisc.ernet.in/arnabde/ opmm-full.pdf.

[7] JSR-133 expert group. Jsr-133: Java memory model and thread specification, August 2004.

[8] G. Gopalakrishnan, Y. Yang, and G. Lindstrom. QB or not QB: An efficient execution verification tool for memory orderings. In $C A V, 2004$.

[9] J. Gosling, W. Joy, G. Steele, and G. Bracha. Java(TM) Language Specification, The (3rd Edition) (Java (Addison-Wesley)). Addison-Wesley Professional, 2005.

[10] T.Q. Huynh and A. Roychoudhury. A memory model sensitive checker for C\#. In Formal Methods Symposium (FM), 2006.

[11] T. Lindholm and F. Yellin. Java Virtual Machine Specification. Addison-Wesley Longman Publishing Co., Inc., Boston, MA, USA, 1999.

[12] Jeremy Manson, William Pugh, and Sarita V. Adve. The java memory model. In POPL '05: Proceedings of the 32nd ACM SIGPLAN-SIGACT symposium on Principles of programming languages, pages 378-391, New York, NY, USA, 2005. ACM.

[13] S. Park and D.L. Dill. An executable specification and verifier for relaxed memory order. IEEE Transactions on Computers, 48(2), 1999.

[14] D.C. Schmidt and T. Harrison. Double-checked locking. Pattern languages of program design 3, pages 363-375, 1997.

[15] W. Visser, K. Havelund, G. Brat, and S. Park. Model checking programs. In IEEE international conference on Automated software engineering (ASE), 2000. 